\title{
Impacts of Constraints and Constraint Handling Strategies for Multi-Objective Mechanical Design Problems
}

\author{
Cyril Picard \\ Laboratory of Applied Mechanical Design \\ Ecole polytechnique fédérale de Lausanne (EPFL) \\ Neuchâtel, Switzerland \\ cyril.picard@epfl.ch
}

\author{
Jürg Schiffmann \\ Laboratory of Applied Mechanical Design \\ Ecole polytechnique fédérale de Lausanne (EPFL) \\ Neuchâtel, Switzerland \\ jurg.schiffmann@epfl.ch
}

\begin{abstract}
Multi-objective optimization tools are becoming increasingly popular in mechanical engineering and allow decision-makers to better understand the inevitable trade-offs. Mechanical design problems can however combine properties that make the use of optimization more complex: (i) expensive cost functions; (ii) discrete or step-like behavior of the cost functions; and (iii) non-linear constraints. The latter in particular has a great impact on the convergence and the diversity of the obtained Pareto front.

In this paper, we present five bi-objective mechanical design optimization problems with various levels of constraint complexity. They are used to rigorously benchmark two common constraint handling strategies (constrained-dominance and penalty function). The results suggest that both strategies have similar performance, and that as constraints become more intricate, convergence to the best-known Pareto front is not guaranteed. Indeed, analyzing the evolution of the hypervolume along generations reveals that the optimizer can get trapped in local optima. A detailed analysis of the obtained Pareto fronts for the proposed problems allows us to qualify the effects of the different constraints.
\end{abstract}

\section{CCS CONCEPTS}

- Applied computing $\rightarrow$ Engineering; • Theory of computation $\rightarrow$ Evolutionary algorithms;

\section{KEYWORDS}

Mechanical engineering, Constraint handling, Multi-objective optimization, Performance measures

\section{ACM Reference Format:}

Cyril Picard and Jürg Schiffmann. 2018. Impacts of Constraints and Constraint Handling Strategies for Multi-Objective Mechanical Design Problems. In GECCO '18: Genetic and Evolutionary Computation Conference, fuly 15-19, 2018, Kyoto, fapan. ACM, New York, NY, USA, 7 pages. https: //doi.org/10.1145/3205455.3205526

\section{INTRODUCTION}

The design of mechanical systems is often an intrinsically conflicting task. Ideal solutions rarely exist and engineers are forced to make compromises (weight over strength, price over performance).

GECCO '18, fuly 15-19, 2018, Kyoto, Japan

(C) 2018 Copyright held by the owner/author(s). Publication rights licensed to Association for Computing Machinery.

This is the author's version of the work. It is posted here for your personal use Not for redistribution. The definitive Version of Record was published in GECCO '18: Genetic and Evolutionary Computation Conference, Fuly 15-19, 2018, Kyoto, Japan https://doi.org/10.1145/3205455.3205526.
The formulation of a design task as a multi-objective problem (MOP) is thus straightforward. Indeed, multi-objective optimization (MOO) is gradually gaining importance in mechanical engineering $[10,28,29,31]$, as it helps gain useful insights into the problem at hand and increases the decision-making capabilities of engineers. In the MOO community, engineering inspired applications have often been used as examples [3, 7, 26].

Practically though, the objective functions of real-world mechanical MOPs can rarely be expressed as algebraic equations and are often the result of simulation-based models. As such, running an optimization process is a computationally expensive task. Constraints imposed by the problem can also be the outcome of simulations and their mathematical behavior is often unknown beforehand and all sorts of non-linearity can be expected. In addition, step-like or discrete behavior of both the objectives and the constraints can be assumed, as mechanical design almost always implies categorical choices.

In order to solve such MOPs, stochastic metaheuristics and in particular multi-objective evolutionary algorithms (MOEAs), have been successfully applied for the past decades and proved to be powerful global search algorithms.

Nature of the issue. Complex real-world mechanical design problems have features that are challenging for MOEA. In particular most problems are constrained MOP, formally defined by equation (1). While test suites commonly used to benchmark the performance of new algorithms now also feature constrained problems, e.g. CF from the CEC 2009 competition [34] and C-DTLZ [21], most MOEA have not been specifically designed for constrained problems.

$$
\begin{array}{cl}
\min & \left(f_{1}(x), f_{2}(x), \ldots, f_{m}(x)\right) \\
\text { subject to } & g_{j}(x) \geq 0, \quad j=1,2, \ldots, p \\
& h_{k}(x)=0, \quad k=1,2, \ldots, q \\
& x_{i}^{(L)} \leq x \leq x_{i}^{(U)}, \quad i=1,2, \ldots, n
\end{array}
$$

Box constraints can be handled directly by the selected variation operators, but inequality and equality constraints need to be taken care of by means of a constraint handling strategy. Different constraint handling strategies have been proposed [2, 21, 22, 32], but selection guidelines and the practical consequences for engineers are scarcely present in literature.

Goals and objectives. The design of an electro-mechanical actuator is considered and a set of five problems are specifically built to characterize and assess different types of constraints and their effects on the optimization process. The goal is to investigate on this 
problem set two simple and common constraint handling strategies that are readily available in NSGA-II. The objectives are to identify the most performing strategy depending on the nature of the constraints and to understand how the convergence process is affected.

Scope of the paper. The concepts of the considered engineering problem are presented in Section 2 along with the proposed benchmark problems. Their specifications and expected level of difficulty are discussed. Section 3 describes the optimizer used to solve these problems and a brief review of existing constraint handling strategies is made. The experimental conditions are clarified. The results of the performed benchmark are reported in Section 4 and practical observations and their consequences are discussed.

\section{MULTI-OBJECTIVE DESIGN OF ELECTRO-MECHANICAL ACTUATORS}

Electro-mechanical actuators are devices mainly composed of an electric motor and a gearbox wrapped in a housing that holds the components in place and protects them from the environment. These devices are often integrated in systems where position control or motion is required.

Although seemingly simple, the design process of these systems can be tedious, given electric motor, gearbox and housing are closely coupled and need to be matched in order to fulfill the required output speed and output torque, while satisfying constraints such as cost, volume, weight and efficiency.

\subsection{Decision variables and modeling}

Following a generic approach, the whole actuator is modeled as a set of smaller sub-models that are connected together. The integrated model provides information on the actuator performance, its cost and 3D layout details, Figure 1.

The electric motor is selected among a list of existing stepper motors and its coils can be scaled using a fill-factor $(F F)$ coefficient. Its performance is predicted following Acarnley [1] and its cost is based on existing products and adjusted to the selected $F F$. Simplified mesh information is used for the 3D representation.

The gearbox is modeled as $k$ stages of spur-gear pairs where the wheel of a stage is rigidly connected to the pinion of the next stage. Each spur-gear pair is represented by 6 design variables: the module $m_{i}$ and the thickness to module ratio $b_{i}$ of the stage, the number of teeth $Z_{i(1,2)}$ and the profile-shift coefficient $x_{i(1,2)}$ for pinion and wheel. 3D layout information of a pair is given by two additional design variables: the axial shift from the previous stage $d_{i}$ and the relative angular position of the wheel with respect to the pinion $\gamma_{i}$, as proposed by [31]. Calculations on the gear train are performed using the geometrical, kinematic and strength relations from ISO norms [15-19] and following recommendations from $[25,30]$. An approximate cylinder is used as 3D mesh information and its volume is used to compute the cost.

Finally, the housing is modeled by a convex hull with a fixed thickness wrapped around the motor and gearbox and only contributes to the cost.
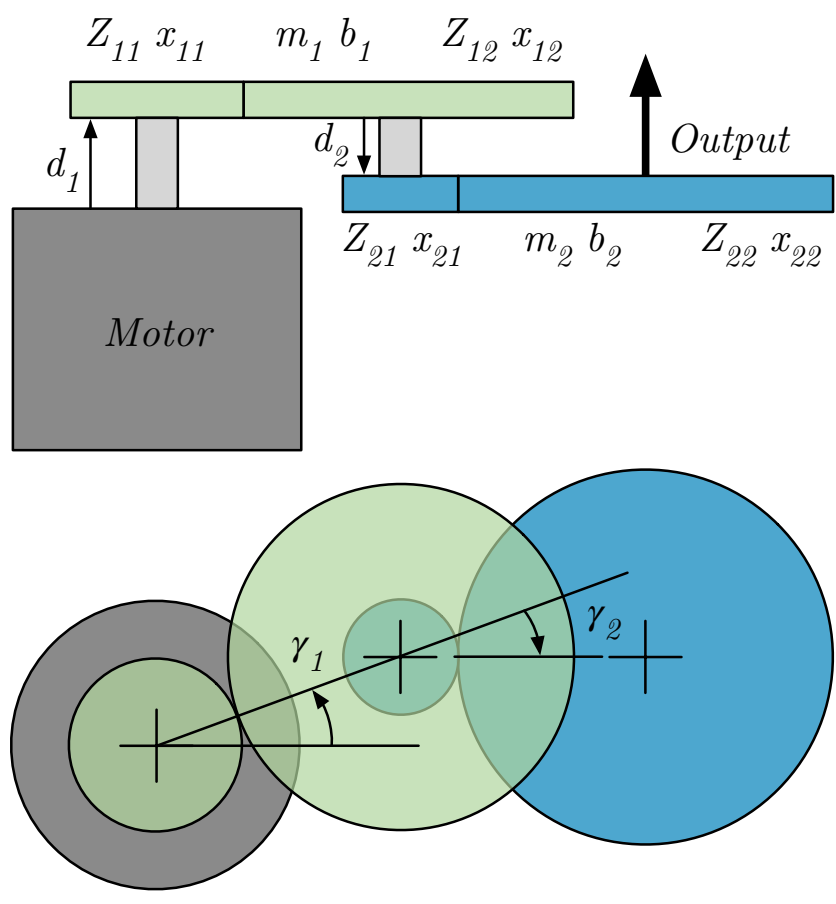

Figure 1: Schematic representation of an electro-mechanical actuator with indications on the design variables.

\subsection{Objectives and constraints}

The design of a particular actuator can have many different types of objectives. As our focus is set on the effects of constraints, a bi-objective case is considered, where the total cost is minimized and the torque excess is maximized. The total cost is the sum of the cost for motor, gears and housing. The torque excess is the effective torque at the output for the required speed minus the required torque. While the first objective is often straightforward for industrial applications, the second one gives engineers the possibility to assess the cost landscape around the ideal solution (no excess torque).

The constraints considered are divided into three groups:

Kinematics. In order to ensure proper operation of a given gear stage, tooth profile interference, minimum specific sliding and contact ratio must be checked. These constraints are active only when combining boundary values for the number of teeth and the profileshift coefficient for the pinion and the wheel. They are also independent for each stage of the gearbox.

Strength. The resistance of the gears to the transmitted torque is verified by calculating the stress due to bending and the Hertzian pressure on the flanks of the gear tooth and comparing them to material limits. These constraints not only involve all design variables for a given stage, they are also dependent of previous stages.

$3 D$ layout. The modeling of the layout of the different components in the 3D space needs to satisfy constraints linked to spacial interference, assembly feasibility and general volume requirements. 
The spacial interference measures the normalized number of mesh elements that are in the volume of another part. The assembly feasibility looks for deadlocks in the construction of the system, following work done in [13]. Finally, additional constraints on the total volume can be specified either in terms of maximal volume or bounding box limits.

\subsection{Proposed test problems}

Using the models defined previously, five mechanical design problems are analyzed, which aim at designing an actuator composed of an electric motor and 4 spur-gear stages, while minimizing the total cost and maximizing the excess torque at the output. The proposed models are built with two levels of detail - with and without 3D layout considerations - and with an increasing number of constraints. Table 1 gives a summary of the considered constraints for these problems (LJ1-5).

In addition, the contact ratio, the minimum specific sliding and the strength safety factor should be equal or higher than $1.2,-5$ and 1.5 , respectively. Further, when applying bounding box limits, the actuator should fit inside a box of $40 \times 63 \times 30.5 \mathrm{~mm}^{3}$. The lower and upper bounds for the design variables are given as follow:

$$
\left[\begin{array}{c}
9 \\
20 \\
0.3 \\
5 \\
-0.6 \\
-30 \\
-\pi
\end{array}\right] \leq\left[\begin{array}{c}
Z_{i 1} \\
Z_{i 2} \\
m_{i} \\
b_{i} \\
x_{i,(1,2)} \\
d_{i} \\
\gamma_{i}
\end{array}\right] \leq\left[\begin{array}{c}
80 \\
100 \\
1 \\
15 \\
0.6 \\
30 \\
\pi
\end{array}\right]
$$

The available motors, the operating specifications, the material properties and the cost information are provided by an industrial partner and are kept constant for all problems.

\section{CONSTRAINT HANDLING STRATEGIES}

The optimization of such constrained problems using MOEAs requires the use of a constraint handling strategy. According to Takahama et al. [33], these strategies can be classified into the following categories: (1) death penalty methods, (2) penalty function methods (static or adaptive), (3) extended dominance methods, (4) objectivization methods, and (5) hybrid methods. Category 1 is the easiest to implement: invalid points are assigned an arbitrarily high objective value. Yet, no indication on the level of violation is given to the algorithm, which is not expected to perform well except for convex constraints or for constraints that only have a limited impact on the search space [4]. Categories 2 and 3 use the constraint violation $(C V)$, calculated with equation (3), to either penalize the objective values or to extend the used dominance concept. Objectivization methods include the constraints as one or several additional objectives in the optimization process, creating a many-objective optimization problem. As such, solving the resulting problem could be significantly more difficult [11, 20]. Hybrid methods combine aspects of the previous categories and offer good performance perspectives. Yet, most of them are not ready to be used by practitioners from other fields.

$$
C V(x)=\sum_{j=1}^{p}\left|\min \left(g_{j}(x), 0\right)\right|+\sum_{k=1}^{q}\left|h_{k}(x)\right|
$$

Penalty function and extended dominance methods are commonly used in practice and are the focus of this paper.

\subsection{Penalty functions}

Penalty function methods add a penalty to the objective values of solutions that violate constraints based on the "amount" of the violation [2]. Typically, the "amount" is measured by the constraint violation $C V$ and is conditioned by means of weights. The new fitness assignment is shown in equation (4), where $Q$ is a function of $C V$ and some weights $w$. The problem (1) is then rewritten as (5).

$$
\begin{array}{r}
f_{i}^{*}(x)=f_{i}(x)+Q(C V(X), w), \quad i=1,2, \ldots, m \\
\text { with } Q \geq 0 \text { and } Q=0 \Longleftrightarrow C V(x)=0 \\
\min \quad\left(f_{1}^{*}(x), f_{2}^{*}(x), \ldots, f_{m}^{*}(x)\right) \\
\text { subject to } \quad x_{i}^{(L)} \leq x \leq x_{i}^{(U)}, \quad i=1,2, \ldots, n
\end{array}
$$

The selection of an appropriate penalty function and its corresponding weights is known to be difficult and problem dependent $[2,27]$. Yet, it has an influence on the convergence process as it will distort the fitness landscape. Depending on the selected algorithm, the added penalty can be misleading for the selection or mating process.

In order to alleviate these issues, adaptive methods have been proposed, e.g. [2, 22]. Yet, for practical applications, adaptive methods are often not readily available and proper tuning of the penalty parameters can be extremely expensive. For these reasons, it is still common to use penalties linear - equation (6) - or quadratic with respect to $C V$ with a single weight $w$.

$$
f_{i}^{*}(x)=f_{i}(x)+w C V(x), \quad i=1,2, \ldots, m
$$

\subsection{Extended dominance}

An alternate solution is to replace the dominance relation used in MOEAs by a dominance relation that takes the constraint violation into consideration. The constrained-dominance [5] and the $\epsilon$ constrained method [32] are two such relations.

The first is the parameterless operator used in NSGA-II [9] and in the more recent NSGA-III [6, 21]. It extends the Pareto dominance as follow:

Definition 3.1. Given two solutions $x_{1}$ and $x_{2}, x_{1}$ is said to constrained-dominate $x_{2}$, if one of the following is true:

(1) $x_{1}$ is feasible and $x_{2}$ is not;

(2) $x_{1}$ and $x_{2}$ are infeasible and $C V\left(x_{1}\right)<C V\left(x_{2}\right)$;

(3) $x_{1}$ and $x_{2}$ are feasible and $x_{1}$ (Pareto-)dominates $x_{2}$.

The $\epsilon$ constrained method is based on the $\epsilon$-dominance [24] and follows the same lexicographic ordering as the constraineddominance method, but includes relaxation of the constraints by means of the $\epsilon$ parameter. 
Table 1: Summary of the proposed mechanical design problems.

\begin{tabular}{lcccl}
\hline & $n$ & $p$ & Cost model & Used constraint groups \\
\hline LJ1 & 17 & 4 & motor and gears & kinematic \\
LJ2 & 17 & 6 & motor and gears & kinematic and strength \\
LJ3 & 25 & 6 & motor, gears and housing & kinematic, space interference and assembly feasibility \\
LJ4 & 25 & 8 & motor, gears and housing & kinematic, strength, spacial interference and assembly feasibility \\
LJ5 & 25 & 11 & motor, gears and housing & kinematic, strength, spacial interference, assembly feasibility and bounding box limits \\
\hline
\end{tabular}

Table 2: Parameters used to configure each run of NSGA-II.

\begin{tabular}{lc}
\hline Parameter & \\
\hline Population size $\mu$ & 120 \\
Function budget & $60^{\prime} 000$ calls \\
& or 500 generations \\
Mutation $\eta_{m}$ & 20 \\
Mutation rate & $1 / n$ \\
Crossover $\eta_{c}$ & 20 \\
Crossover probability (CXPB) & 0.9 \\
\hline Penalty coefficient $w$ & $10,10^{2}, 10^{3}, 10^{6}$ \\
\hline
\end{tabular}

\section{EXPERIMENTAL STUDY}

An experimental study has been conducted using the five proposed problems to benchmark the performance of two constraint handling strategies: the constrained-dominance (called Dominance) and the penalty function defined by equation (6) (called Penalty). In addition, the aggregation of all optimization results is used to obtain approximate Pareto fronts for each problem and to give some insights into the effects of the constraints on the design trade-offs.

\subsection{Experimental setup}

The chosen constraint handling strategies are implemented on top of NSGA-II. The dominance strategy is the default approach for this algorithm, while the penalty strategy is implemented by adapting the objective function. The latter strategy was run with 4 different penalty weights, chosen by progressively decreasing the weight and rerunning the experiment until infeasible solutions become optimal.

The implementation of the considered problems uses continuous variables only. Thus, the traditional variation operators of NSGA-II, namely simulated binary crossover (SBX) and polynomial bounded mutation (PBM) are used. Each problem-strategy combination is run 30 times, configuring the optimizer with the parameters shown in Table 2. The number of function calls is used as termination criterion.

The performance assessment follows best practice from the field [36] and is done using the hypervolume indicator [35] (implementation of Fonseca et al. [12]) with reference point chosen at $(11,11)$. The main advantages of the hypervolume are that it combines convergence and diversity in the objective space in a single metric and that it does not need to know the optimal Pareto front. Yet, two practical aspects need to be taken care of: (i) invalid individuals

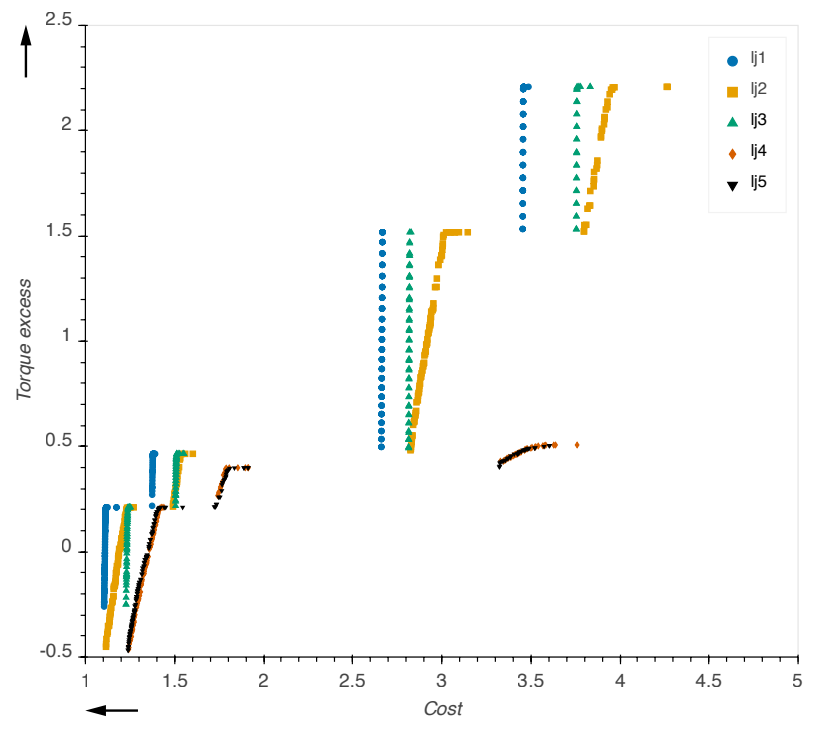

Figure 2: Best-known Pareto fronts for all five test problems.

are removed from the population before the indicator value is computed to ensure that penalty values or unrealistic individuals do not affect the measure; (ii) the second objective (Torque excess) is negated as the hypervolume is designed for minimization only.

The null hypothesis that all strategies are equally well suited is assessed using the non-parametric Kruskal-Wallis test [23] with a confidence interval of $99 \%$.

\subsection{Best-known Pareto fronts}

The best-know Pareto fronts for each problem are shown in Figure 2 and give some indications on the complexity of the proposed problems and on the effects of the different constraints.

In terms of shape, all problems have discrete fronts and show a step-like behavior of the objective functions. Given the discrete nature of the motor selection process, this is an expected result. Nonetheless, spreading the solutions along such steps is known to be difficult, as most density measures used by MOEAs to maintain diversity can be tricked by this kind of shape. Resources are spent trying to fill the step, whereas more exploration might be needed to spread.

The change in modeling level - without and with 3D layout - is also clearly visible in Figure 2 when comparing the fronts of LJ1 with LJ3, and LJ2 with LJ4 and LJ5. It results in a shift of the fronts towards higher cost due to the different cost model. 
In terms of constraints, the following points can be observed:

- The spacial interference and assembly feasibility constraints only play a marginal role on the achievable objectives, as there is no visible difference between the shape of the fronts of LJ1 and LJ3.

- Without strength considerations (LJ1 and LJ3), the torque excess can be increased from 0.5 to 1.5 with barely noticeable effects on cost. In more realistic scenarios (LJ2, LJ4 and LJ5), strength requirements force an increase in size - and thus cost - with increasing torque, resulting in gentler fronts.

- The bounding box constraints (LJ5 compared to LJ4) does not visibly affect the obtained front.

\subsection{Benchmark results and discussion}

The results of the benchmark between the constrained-dominance and the penalty function constraint handling strategies are shown in Table 3. The average hypervolume value as well as its standard deviation for each function and for each strategy are shown. The outcome of the statistical test indicates that there is no statistical difference between the two strategies for all problems and independently of the used penalty weight. It is worth noting that for the lowest weight $(w=10)$, several infeasible solutions were present in the final population. These results can also be seen graphically in Figure 3, where the normalized hypervolume error ${ }^{1}$ is used to allow a better comparison between functions. In addition, Figure 3 gives also an insight into the convergence process. For all problems and independently of the considered strategy, outliers are found and indicate that the algorithm got trapped in a sub-optimal front or it was unable to repeatedly spread along the full Pareto front. The particular shape of the front has already been identified as a source of challenges. Increasing the number of constraints also seems to increase this phenomenon. In particular, the hardest problem LJ5 shows poor convergence: the best-known Pareto front for this problem is achieved by an outlier. Thus, no clear selection rule regarding strategy or penalty weight can be formulated at this point.

Looking at the impact of constraints on convergence, the following statements can be made:

- The more than doubled standard deviation between run results from LJ1 and LJ2 suggests a negative impact on convergence of strength constraints. Indeed, these constraints couple almost all design variables together - and in a nonlinear manner, and are as such expected to be difficult.

- The bounding box constraints, which only have a limited impact on the Pareto front, make the optimization process much harder, as shown by the lower mean and the higher variance of the achieved hypervolume values (LJ5 compared to LJ4).

When discussing convergence issues, the question of the allowed optimization budget is important. In order to assess whether the budget is sufficient for such problems, the analysis of the evolution of the hypervolume along generations can be used as an indicator. For the easiest problem, LJ1 (Figures 4), all runs have converged to a very narrow band within 160 generations. Problem LJ2, Figure 5, reaches a steady value within 220 generations, but the variance

$\left.\overline{{ }^{1} n H V(X)=(H V}\left(P F^{*}\right)-H V(X)\right) / H V\left(P F^{*}\right)$, where $P F^{*}$ is the best-known Pareto front

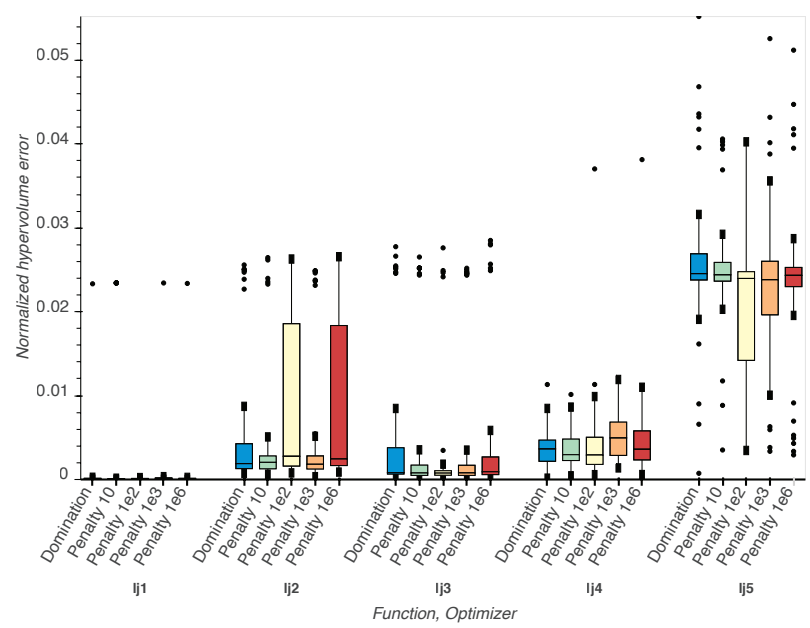

Figure 3: Box plot of the normalized hypervolume error over all runs for the different problems and strategies (lower is better).

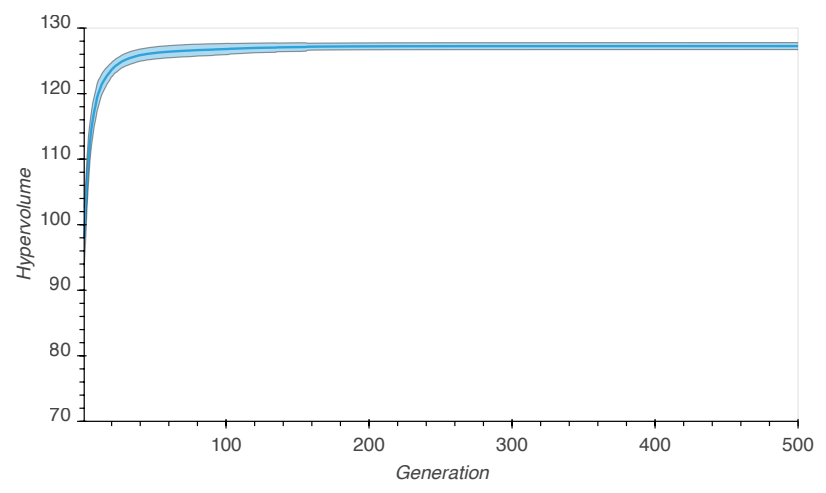

Figure 4: Evolution of the mean and standard deviation of the hypervolume along generations over all runs for problem LJ1.

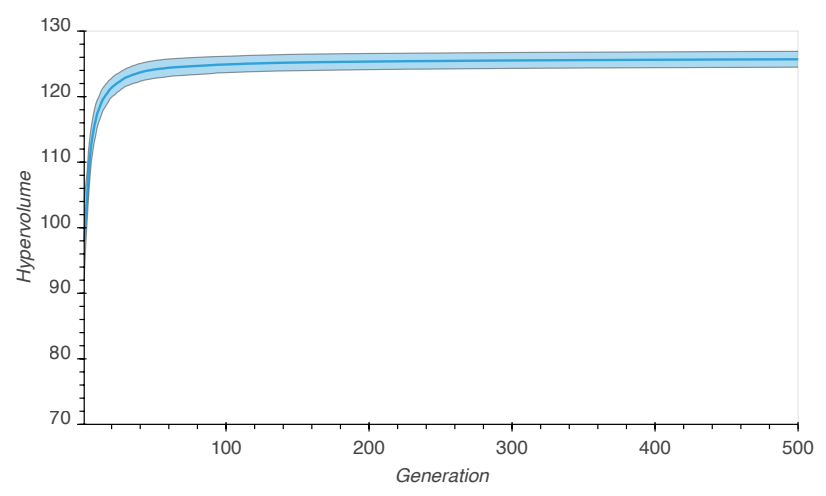

Figure 5: Evolution of the mean and standard deviation of the hypervolume along generations over all runs for problem LJ2. 
Table 3: Mean and standard deviation over all runs of the hypervolume for each test problem.

\begin{tabular}{lrllll}
\hline & Domination & Penalty $w=10^{6}$ & Penalty $w=10^{3}$ & Penalty $w=10^{2}$ & Penalty $w=10$ \\
\hline LJ1 & $127.251 \pm 0.5403$ & $127.251 \pm 0.5413$ & $127.248 \pm 0.5421$ & $127.351 \pm 0.0062$ & $127.056 \pm 0.9082$ \\
LJ2 & $125.799 \pm 1.1711$ & $125.595 \pm 1.2788$ & $125.826 \pm 1.1613$ & $125.566 \pm 1.2830$ & $125.902 \pm 1.1035$ \\
LJ3 & $124.734 \pm 1.3378$ & $124.810 \pm 1.3206$ & $124.887 \pm 1.2269$ & $125.073 \pm 1.0708$ & $124.872 \pm 1.2493$ \\
LJ4 & $111.449 \pm 0.2542$ & $111.315 \pm 0.7414$ & $111.313 \pm 0.2984$ & $111.342 \pm 0.7433$ & $111.493 \pm 0.2338$ \\
LJ5 & $108.921 \pm 1.2813$ & $109.313 \pm 1.3944$ & $109.257 \pm 1.2223$ & $109.452 \pm 1.1069$ & $109.064 \pm 0.9238$ \\
\hline
\end{tabular}

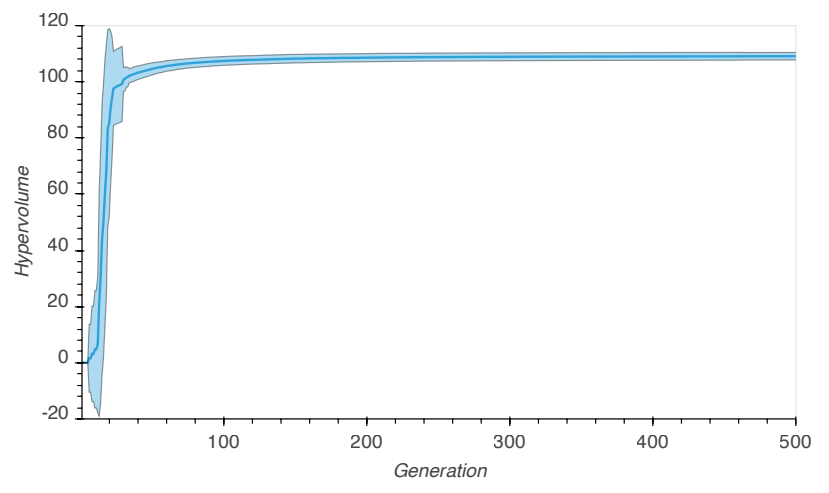

Figure 6: Evolution of the mean and standard deviation of the hypervolume along generations over all runs for problem LJ5. Note the difference in scale of the $y$-axis compared to Figures 4 and 5.

remains high and constant for the remaining generations. This emphasizes that the previously mentioned effects are not due to a lack of budget and that the algorithm is indeed stuck. Similar conclusions can be reached for problem LJ5, Figure 6 .

In addition, the consequences of the reduced valid search space for LJ5 can also be seen in Figure 6: the random initial sampling did not yield any valid solutions and the algorithm needed a few generations to find solutions with no constraint violation. Optimizers lacking specific selection strategies to handle this case face the risk of an early and critical drop in diversity that will affect the ability of the optimizer to spread and to converge. For this particular case, the use of relaxation schemes will be further investigated. A hint of the effect on diversity is highlighted in Figure 7 where the values of the design variables of LJ5 for the population of the worst run and for the best-known front are compared. The difference in diversity between the two is clearly visible and is the consequence of all the challenges previously discussed and combined in LJ5.

\section{CONCLUSIONS}

In this paper, the impacts of constraints and two common constraint handling strategies on the performance of NSGA-II for a set of real-world based mechanical problems have been investigated. In particular, the integrated design of an electro-mechanical actuators has been discussed and five specific formulations of the problem have been proposed with the aim to distinguish the effects of different types of constraints. The experimental study concluded that, independently of the constraints (amount or type), neither the
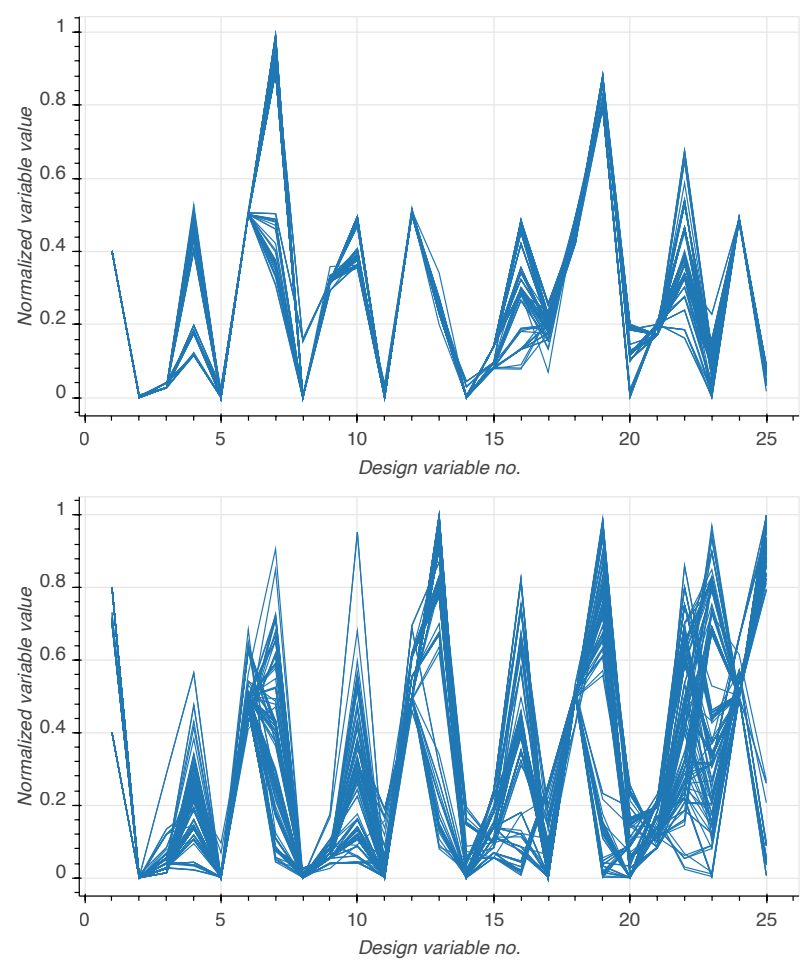

Figure 7: Value path plot for the design variable values of the worse performing run (top) and for the best-known Pareto front (bottom) for LJ5.

constrained-dominance nor the penalty function method performed significantly better. Generally, many runs have shown premature convergence, whose source has been found to be both the step-like behavior of the objective functions and the nature of the constraints. Specifically, constraints coupling many design variables and constraints strongly limiting the search space reduced the performance of the optimizer and undermined the ability of the optimizer to find an appropriate approximation of the optimal Pareto front. For the studied problems, the strength constraints and the bounding box limits were identified as the main constraints affecting the convergence process. The causes are the coupling of all design variables for the first and the search space reduction for the second.

Future work will investigate the development of constraint specific variation operators and diversity improving mechanisms, as well as the use of other constraint handling strategies including relaxation schemes with the aim of finding a more robust optimizer 
for the considered application. Possible starting points are the algorithms built around the $\epsilon$ dominance concept such as $\epsilon$-MOEA [8] or Borg [14].

\section{ACKNOWLEDGMENTS}

The authors would like to thank Dr. Enno de Lange, Dr. Mathieu Lu-Dac and Ing. Paolo Nidola from Johnson Electric International AG for their support and for providing this particular use case. The authors would also like to thank the anonymous referees for their valuable comments and helpful suggestions.

This work is sponsored by the Commission for Technology and Innovation (CTI) of Switzerland

\section{REFERENCES}

[1] Paul Acarnley. 2002. Stepping Motors: A Guide to Theory and Practice. IET.

[2] Carlos A. Coello Coello. 2000. Use of a self-adaptive penalty approach for engineering optimization problems. Computers in Industry 41, 2 (March 2000), 113-127.

[3] Carlos A. Coello Coello, Gary B. Lamont, and David A. Van Veldhuizen. 2007. Evolutionary algorithms for solving multi-objective problems (2. ed ed.). Springer, New York, NY.

[4] Dipankar Dasgupta and Zbigniew Michalewicz. 1997. Evolutionary Algorithms in Engineering Applications. Springer, Berlin.

[5] Kalyanmoy Deb. 2001. Multi-Objective Optimization Using Evolutionary Algorithms. John Wiley \& Sons.

[6] K. Deb and H. Jain. 2014. An Evolutionary Many-Objective Optimization Algorithm Using Reference-Point-Based Nondominated Sorting Approach, Part I: Solving Problems With Box Constraints. IEEE Transactions on Evolutionary Computation 18, 4 (Aug. 2014), 577-601.

[7] Kalyanmoy Deb and Sachin Jain. 2003. Multi-Speed Gearbox Design Using MultiObjective Evolutionary Algorithms. Fournal of Mechanical Design 125, 3 (2003), 609

[8] Kalyanmoy Deb, Manikanth Mohan, and Shikhar Mishra. 2005. Evaluating the $\varepsilon$-Domination Based Multi-Objective Evolutionary Algorithm for a Quick Computation of Pareto-Optimal Solutions. Evolutionary Computation 13, 4 (Dec. 2005), 501-525.

[9] K. Deb, A. Pratap, S. Agarwal, and T. Meyarivan. 2002. A fast and elitist multiobjective genetic algorithm: NSGA-II. IEEE Transactions on Evolutionary Computation 6, 2 (April 2002), 182-197.

[10] Kalyanmoy Deb and Santosh Tiwari. 2005. Multi-objective optimization of a leg mechanism using genetic algorithms. Engineering Optimization 37, 4 (June 2005), 325-350.

[11] M. Farina and P. Amato. 2002. On the optimal solution definition for manycriteria optimization problems. In 2002 Annual Meeting of the North American Fuzzy Information Processing Society Proceedings. NAFIPS-FLINT 2002 (Cat. No. 02TH8622). 233-238.

[12] C. M. Fonseca, L. Paquete, and M. Lopez-Ibanez. 2006. An Improved DimensionSweep Algorithm for the Hypervolume Indicator. 1157-1163.

[13] Pierre M. Grignon and Georges M. Fadel. 2004. A GA Based Configuration Design Optimization Method. Fournal of Mechanical Design 126, 1 (March 2004), 6-15.

[14] David Hadka and Patrick Reed. [n. d.]. Borg: An Auto-Adaptive Many-Objective Evolutionary Computing Framework. 21, 2 ([n. d.]), 231-259.

[15] International Organization for Standardization. 1998. Cylindrical gears for general and heavy engineering - Standard basic rack tooth profile. ISO 53:1998. Geneva Switzerland. https://www.iso.org/standard/22643.html

[16] International Organization for Standardization. 1998. Vocabulary of gear terms - Part 1: Definitions related to geometry. ISO 1122-1:1998. Geneva, Switzerland. https://www.iso.org/standard/5649.html
[17] International Organization for Standardization. 2006. Calculation of load capacity of spur and helical gears. ISO 6336:2006. Geneva, Switzerland. https://www.iso. org/standard/36329.html

[18] International Organization for Standardization. 2007. Gears - Cylindrical involute gears and gear pairs - Concepts and geometry. ISO 21771:2007. Geneva, Switzerland. https://www.iso.org/standard/35989.html

[19] International Organization for Standardization. 2013. Cylindrical gears - ISO system of flank tolerance classification. ISO 1328-1:2013. Geneva, Switzerland. https://www.iso.org/standard/45309.html

[20] H. Ishibuchi, N. Akedo, and Y. Nojima. 2015. Behavior of Multiobjective Evolutionary Algorithms on Many-Objective Knapsack Problems. IEEE Transactions on Evolutionary Computation 19, 2 (April 2015), 264-283.

[21] H. Jain and K. Deb. 2014. An Evolutionary Many-Objective Optimization Algorithm Using Reference-Point Based Nondominated Sorting Approach, Part II: Handling Constraints and Extending to an Adaptive Approach. IEEE Transactions on Evolutionary Computation 18, 4 (Aug. 2014), 602-622.

[22] M. A. Jan and Q. Zhang. 2010. MOEA/D for constrained multiobjective optimization: Some preliminary experimental results. In 2010 UK Workshop on Computational Intelligence (UKCI). 1-6.

[23] William H. Kruskal and W. Allen Wallis. 1952. Use of Ranks in One-Criterion Variance Analysis. F. Amer. Statist. Assoc. 47, 260 (Dec. 1952), 583-621.

[24] Marco Laumanns, Lothar Thiele, Kalyanmoy Deb, and Eckart Zitzler. 2002. Combining Convergence and Diversity in Evolutionary Multiobjective Optimization. Evolutionary Computation 10, 3 (Sept. 2002), 263-282.

[25] Gustav Niemann and Hans Winter. 2003. Getriebe allgemein, Zahnradgetriebe - Grundlagen, Stirnradgetriebe (2., völlig neubearb. aufl., 2. berichtigter nachdr., korrigierter nachdr ed.). Number G. Niemann; H. Winter ; Bd. 2 in Maschinenelemente. Springer, Berlin.

[26] Singiresu S. Rao. 2009. Engineering Optimization: Theory and Practice. John Wiley \& Sons.

[27] Jon T. Richardson, Mark R. Palmer, Gunar E. Liepins, and Mike Hilliard. 1989. Some Guidelines for Genetic Algorithms with Penalty Functions. In Proceedings of the Third International Conference on Genetic Algorithms. Morgan Kaufmann Publishers Inc., San Francisco, CA, USA, 191-197.

[28] Jérémi Régnier, Bruno Sareni, Xavier Roboam, and Stéphan Astier. 2003. Optimal design of electrical engineering systems using Pareto Genetic Algorithms.

[29] J. Schiffmann. 2015. Integrated Design and Multi-objective Optimization of a Single Stage Heat-Pump Turbocompressor. Fournal of Turbomachinery 137, 7 (July 2015), 071002-071002-9.

[30] Joseph Edward Shigley and Charles R. Mischke. 2001. Mechanical engineering design (6th ed ed.). McGraw Hill, Boston, Mass.

[31] Albert Swantner and Matthew I. Campbell. 2012. Topological and parametric optimization of gear trains. Engineering Optimization 44, 11 (Nov. 2012), 13511368.

[32] Tetsuyuki Takahama and Setsuko Sakai. 2005. Constrained Optimization by $\varepsilon$ Constrained Particle Swarm Optimizer with $\varepsilon$-level Control. In Soft Computing as Transdisciplinary Science and Technology (Advances in Soft Computing). Springer, Berlin, Heidelberg, 1019-1029.

[33] Tetsuyuki Takahama and Setsuko Sakai. 2015. Efficient Constrained Optimization by the epsilon Constrained Differential Evolution with Rough Approximation. In Evolutionary Constrained Optimization, Rituparna Datta and Kalyanmoy Deb (Eds.). Springer India, 157-180.

[34] Qingfu Zhang, Aimin Zhou, Shizheng Zhao, Ponnuthurai Nagaratnam Suganthan, Wudong Liu, and Santosh Tiwari. 2008. Multiobjective optimization test instances for the CEC 2009 special session and competition. University of Essex, Colchester, UK and Nanyang technological University, Singapore, special session on performance assessment of multi-objective optimization algorithms, technical report 264 (2008).

[35] E. Zitzler and L. Thiele. 1999. Multiobjective evolutionary algorithms: a comparative case study and the strength Pareto approach. IEEE Transactions on Evolutionary Computation 3, 4 (Nov. 1999), 257-271.

[36] E. Zitzler, L. Thiele, M. Laumanns, C. M. Fonseca, and V. G. da Fonseca. 2003. Performance assessment of multiobjective optimizers: an analysis and review. IEEE Transactions on Evolutionary Computation 7, 2 (April 2003), 117-132. 